\title{
Short Range Inter-satellite Link for Data Transfer and Ranging using IEEE802.11n
}

\author{
Viraj Shah \\ Master of Technology \\ Department Of \\ Computer Engineering \\ CSPIT, Charusat
}

\author{
Ritesh Patel \\ Associate Professor \\ Department Of \\ Computer Engineering \\ CSPIT, Charusat
}

\author{
Rikin Nayak \\ Assistant Professor \\ CHARUSAT Space Research \\ Technology Center (CSRTC) \\ CSPIT, Charusat
}

\begin{abstract}
Satellites are specifically made for telecommunication purpose. In satellite systems, On-Board communication is a one of the key aspect in which transfer of digital data (high speed high volume data) \& TM-TC (Tele command or Telemetry, low volume low speed data) is taking place. Data communication among satellite systems also known as InterSatellite Communication Link provides connectivity among two or more satellites and eliminating the need for intermediate ground stations when communicating data. Inter satellite links have been considered for satellite constellation missions involving earth observation and communications. In this paper, the feasibility of IEEE 802.11n standard for Intersatellite communication and Ranging between two Wi-Fi enable devices has discussed.
\end{abstract}

\section{Keywords}

Inter-satellite link, Wi-Fi Direct, IEEE 802.11, Ranging.

\section{INTRODUCTION}

A Low Earth Orbit (LEO) is an orbit about $160 \mathrm{~km}$ to $2000 \mathrm{~km}$ above earth's surface, and a time taken to rotate around the earth is about 90 minutes. From the sub satellite point, this satellite are visible only from $1000 \mathrm{~km}$. Satellite in LEO can change their position quickly related to ground station so for the uninterrupted connectivity large number of satellites are needed. LEO satellite has less distance from the earth's surface so it is less expensive then the Medium Earth Orbit (MEO) \& Geostationary Earth Orbit (GEO) satellites. LEO satellite does not require very high signal strength. Due to the proximity to Earth, LEO satellites have a lower latency and require less amplification for transmission. In LEO Satellite Transmitting station is not as much powerful as MEO \& GEO. LEO Satellites are mainly used for data communication, military purpose, aeronautical purpose etc. The LEO based satellite can provide 3\% access of the Earth's surface at any given time. The Life span of LEO Satellite is about 5 years. LEO satellite constellations provides an advantages over GEO satellite, it take $125 \mathrm{~ms}$ for a signal to get from ground to GEO, where it takes 1-4ms for LEO. LEO also provides more system capacity by frequency reuse across its coverage.

The WI-FI includes two spread-spectrums radio techniques 1) frequency hopping spread spectrums (FHSS) 2) direct sequence spread spectrum (DSSS). Using FHSS, $2.4 \mathrm{GHz}$ band is split into 75 sub channels of $1 \mathrm{MHz}$. Both sender and receiver are agreed on the hopping scheme, and data is directed to the sub channels. This technique grant for a corresponding simple radio design, but are bounded to bandwidth of not more than 2 Mbps. On other hand, DSSS splits $2.4 \mathrm{GHz}$ band into fourteen $22 \mathrm{MHz}$ sub channels. Bordering channels lap over one another fractionally, with 3 of the 14 being completely non-overlaying. IEEE802.11 uses a somewhat modified protocol known as Carrier Sense Multiple Access with Collision Avoidance (CSMA/CA). CSMA/CA avoid collisions by using explicit packet acknowledgment (ACK), which means an acknowledgement packet is sent by the receiving station to confirm that the data packet arrived intact. In this paper, wireless technology that has been used is Wireless-Fidelity (WI-FI). WI-FI is a Technology that allows any wifi enables computers or wifi enable devices to communicate over wireless signals. It represents network components that are based on one of the 802.11 standards developed by IEEE and adopted by WI-FI Alliance. IEEE802.11 wireless networks can be configured into two different modes: ad hoc and infrastructure modes. In ad hoc mode, all wireless stations within the communication range can communicate directly with each other, whereas in infrastructure mode: an Access Point (AP) is used to connect all stations to a Distribution System (DS), and each station call communicate with others through the AP. A Wi-Fi is a data transmission system designed to provide locationindependent network access between computing devices by using radio waves rather than a cable infrastructure.

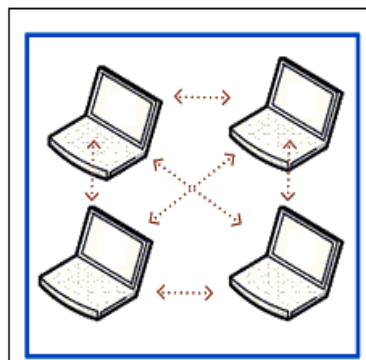

Ad-hoc mode

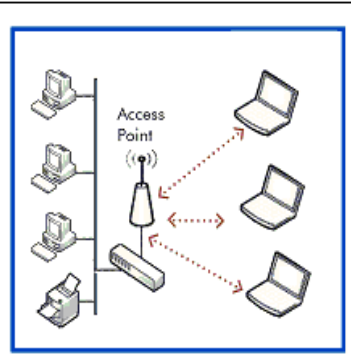

Infrastructure mode
Figure 1: Ad-hoc mode vs. Infrastructure mode

IEEE802.11 introduced many types of the Wi-Fi standard. These have all different transmission bandwidth form one transmitting node to other receiver node. Radio frequency is $2.4 \mathrm{GHz}$ or $5 \mathrm{GHz}$. The Modulation technique of radio frequency is increases the bandwidth of data. There is various type of the Wi-Fi standard but most commonly standards are $802.11 \mathrm{a} / \mathrm{b} / \mathrm{g} / \mathrm{n} / \mathrm{ac}$ etc. There are so many wireless technologies available like Bluetooth, Zigbee, WI-FI etc. According to the comparison of Wireless-Fidelity, Bluetooth and Zigbee, the WI-FI is best technology for wireless communication. Related to the bandwidth of data transmission, WI-FI has higher bandwidth than the Bluetooth and Zigbee \& their bandwidth are $11 \mathrm{Mbps}$ to $600 \mathrm{Mbps}, 723 \mathrm{Kbps}$ and $250 \mathrm{Kbps}$ respectively. WI-FI supports the dual band $2.4 \mathrm{GHz} \& 5 \mathrm{GHz}$ related to the dual band radio frequency. The data transmission range is $100 \mathrm{~m}$ for Bluetooth and Zigbee 
facilitates with short range and low data rate communication. Due to the high-speed transmission, the power consumption of the WI-FI is more as compared to the Bluetooth and Zigbee. Thus, WI-FI is best option to communicate for inter satellites.

Table 1: comparison of different wireless technology

\begin{tabular}{|l|l|l|l|}
\hline PARAMETER & WI-FI & $\begin{array}{l}\text { BLUETOOT } \\
\text { H }\end{array}$ & $\begin{array}{l}\text { ZIGBE } \\
\text { E }\end{array}$ \\
\hline Standard & $\begin{array}{l}\text { IEEE802 } \\
11 \mathrm{a} / \mathrm{b} / \mathrm{g} / \\
\mathrm{n} / \mathrm{ac}\end{array}$ & IEEE802.15.1 & $\begin{array}{l}\text { IEEE80 } \\
2.15 .4\end{array}$ \\
\hline RF band & $\begin{array}{l}2.4 \mathrm{GHz} \\
\text { or } 5 \mathrm{GHz}\end{array}$ & $2.4 \mathrm{GHz}$ & $2.4 \mathrm{GHz}$ \\
\hline Data rate & $\begin{array}{l}11 \mathrm{mbps} \\
\text { to } \\
600 \mathrm{mbps}\end{array}$ & $723 \mathrm{kbps}$ & $\begin{array}{l}250 \\
\mathrm{kbps}\end{array}$ \\
& $\begin{array}{l}\text { Up to } \\
150 \mathrm{~m}\end{array}$ & $100 \mathrm{~m}$ & $\begin{array}{l}\text { Up to } \\
10 \mathrm{~m}\end{array}$ \\
\hline Range & High & Low & very low \\
\hline Power & \multicolumn{2}{|l}{}
\end{tabular}

\section{RELATED WORK}

Satellites are specifically made for telecommunication purpose. The satellite systems are using microwave technology for space-to-ground communication, but satelliteto-satellite communication is carried out by Inter-satellite Link (ISL). For a LEO satellite system there are mainly two inter-satellite links. The first type is the link between satellites on the same orbital plane, called intraorbit inter-satellite link. The second type is link between satellites on different orbital planes, called interorbit inter-satellite link.

The wireless communication between two LEO based satellite is done by adapting Commercial-of-the-Shelf (COTS) wireless standard. In COTS approach the physical layer \& Data-link layer parameter has been changed for the Space Application. The criteria of the selection of wireless protocol for intra-satellite links are based on robustness and fault tolerance. Same way, the criteria of the selection of wireless protocol for inter-satellite links are based on Range, networking feature, power requirements and mobility. As investigated Bluetooth \& Zigbee are for intra-satellite communication and Wi-Max \& Wi-Fi are for inter-satellite communication. IEEE 802.16e Wi-Max has a feature such as extensive support for Qos, full native IP support, mobility, OFDMA and high physical layer efficiency is very valuable for inter-satellite communications. Whereas IEEE 802.11 WiFi supports Bi-directional antennas and mesh network deployment could be used for inter-satellite communication [1].

To improve the IEEE802.11e protocol performance, new measurement-based method admission control method [2] is used. The aim of any admission control is to ensure that a resource-limited network does not degrade Qos of the network. In admission control method, control function decides that connection can be granted, depending on the amount of the existing traffic load. So, admission control is a key component of any Qos-based resource management scheme.

The signal-to-noise ratio (SNR) is the gold standard metric that captures not only the quality of a wireless link, but also offers limited predictability [3]. In the same SNR the performance of wireless link can be different. Reasons for SNR to fails to predict performance of wireless link is frequency diversity. At the frequency of $20 \mathrm{MHz}$ WI-FI channel can differ significantly. As the traffic demand increases the frequency diversity can be increased.

IEEE802.11n standard is the first IEEE 802.11 improvement to suggest Multiple-Input and Multiple-Output (MIMO) [4] transmission scheme. It provides data rates up to 600Mbits/s. IEEE802.11n provides channel bonding, space-time block coding \& spatial division multiplexing. Channel bonding doubles physical data rate by using two adjacent IEEE802.11a or IEEE $802.11 \mathrm{~b} / \mathrm{g}$. It also covers $40 \mathrm{MHz}$ instead of $20 \mathrm{MHz}$. Spatial division multiplexing and Space-time block coding explains the multi-path propagation. The MIMO feature makes IEEE802.11n encouraging technology for architecturing wireless mesh network. High data rates of IEEE802.11n can only be utilized at upper layer if medium access is competent. IEEE 802.11n introduced frameaggregation. In this method, multiple subframes can be dispatched in sequence with the overhead for medium access originating only once. IEEE $802.11 \mathrm{n}$ standard represents two different variant schemes: aggregated MAC service data unit (A-MSDU) and aggregated MAC protocol data unit (AMPDU) [5]. A-MSDU scheme allows encapsulating several packets in a one MAC frame with a one MAC header and a one frame check sequence (FCS). Receiving node can only investigate the sincerity of the whole A-MSDU frame, hence the whole A-MSDU has to be retransmitted in case of transmission errors. A-MPDU frame can be aggregated by multiple MPDU with a common Physical header. Each subframe size should be in multiple of 4 bytes expected the last sub-frame. Maximum size of A-MPDU frame is maximum 64 $\mathrm{KB}$, which means it can aggregated maximum of 64 MSDUs.

Wi-Fi Direct is technology that aims to connect devices with one another without requiring of wireless access point. It enables the peer-to-peer communication between devices at the same Wi-Fi speed [6]. Essentially Wi-Fi Direct embedded software access point (soft AP) into any device that supports Direct. This soft AP provides a version of $\mathrm{Wi}-\mathrm{Fi}$ protected setup with its push-button or PIN-based setup. Wi-Fi Direct devices conventionally known as $\mathrm{P} 2 \mathrm{P}$ devices, which are usually Wi-Fi infrastructure networks. The device act like AP is referred as Group Owner, and user acts as a Clients. The roles are not static, it can be changed upon the situation. When the group is created the other clients can join the group by typical Wi-Fi network. Wi-Fi devices are enforced to appliance Wi-Fi protected Setup (WPS) to support secure connection. WPS originated a secure connection by PINbased setup or Push-button in two P2P devices. WPS is situated on WPA-2 security and uses Advance Encryption Standard (AES) [7]. Wi-Fi Direct has higher range and bandwidth then Bluetooth and Zigbee which is added an advantage.

\section{INTER SATELLITE COMMUNICATION AND RANGING}

In satellite systems, On-Board communication is a one of the key aspect in which transfer of digital data (high speed high volume data) \& TM-TC (Tele command or Telemetry, low volume low speed data) is taking place. On-Board communication between different subsystems of the satellite is generally carried out using wired interfaces also known as Intra-Satellite Communication. Data communication among satellite systems also known as Inter-Satellite Communication Link provides connectivity among two or more satellites. Inter-satellite links have been considered for satellite constellation missions involving earth observation and communications. Inter-satellite links or cross-links provide 
direct connectivity between two satellites, thus eliminating the need for intermediate ground stations when sending data. Inter-satellite link can also provide advance functionality like Relative navigation and formation control and clock synchronization. Using inter-satellite link bandwidth can be increased and power consumption decreased.

Ranging measures time taken for a signal to reach from sender to receiver. In ranging, it is used to measure the Round Trip Time (RTT) of the signal. Currently, Satellite ranging is the most accurate technique available to determine the geographical position of the satellites. In this method, Receiver calculates distance to satellites as a function of amount of time it takes for satellite signals to reach the ground. To make such a calculation, the receiver must be able to tell accurately when the signal was transmitted and when it was received. The satellites are equipped with extremely accurate atomic clocks, so the timing of transmissions is always known. The signals broadcast by satellites, called "pseudo-random codes," are accompanied by the broadcast ephemeris data that describes the shapes of satellite orbits. There are mainly 3 approaches 1 ) using RSSI value 2) using Time delay 3 ) using pseudorandom.

First Approach is to measure the distance is using the WI-FI signal strength. Wi-Fi signal strength is tricky. The appropriate way to measure signal strength is to measure Received Signal Strength Indicator (RSSI) value [8]. The accuracy of the RSSI can be varying due to devices, implementations and environmental limitations. The RSSI value is measured in $\mathrm{dBm}$. The measurement of the RSSI value is different for all the Wi-Fi devices manufactured by different vendors because they may follow different implementation. With the help of the RSSI value you can measure the approximate distance.

Second approach is to measure the distance is using time delay [9]. In this method, One transmitter will start transmits data to receiver and starts the clock. At the same way when receiver starts receiving the data it will start the clock and send back the "echo" \& the receiving clock time to the transmitter. Then, the transmitter will measure the time difference between transmitted-clock and received-clock. This time will indicates signal delay from transmitter to receiver. With the help of this delay, approximately distance between transmitters to receiver is measured. For the better accuracy the same algorithm is applied on both sides. The receiver may act as transmitter and the transmitter may act as receiver.

Third approach is to measure the distance is using pseudorandom (PRN) code [10]. The PRN code can be generated using maximum length shift register. PRN code can be chip code or GPS code having a well-defined autocorrection function. By filtering and modulating PRN code, the location symbol can be generated. This location symbol is useful in the location packets of wireless devices. Then, the location packets can be sent from one wireless device to another wireless device. After successfully transformation of location packets, the round trip time can be measured. The approximate distance between two wireless devices can be measured using this round trip time.

\section{CONCLUSION}

The purpose of this paper is to investigate the possibility of using IEEE 802.11 Wi-Fi standard for low range Intersatellite communication. By Inter-satellite communication the throughput and bandwidth for down-link can be increased as results requirements of the more numbers of ground-stations can be eliminated. For low range communication Wi-Fi is a suitable standard protocol. IEEE 802.11n standard supports higher data-rate up to $600 \mathrm{mbps}$ and it uses multiple antennas to increase data-rates (MIMO). The standard works in 2.4 $\mathrm{GHz} \& 5 \mathrm{GHz}$ frequency band. For peer-to-peer communication Wi-Fi Direct mode is useful. Wi-Fi Direct devices can connect each other without having gone through an access point so the connection time is less compare to other Wi-Fi modes. With inter-satellite communication using wifi standard the range between two Wi-Fi enable devices can be calculated using 3 approaches 1) using RSSI value 2) using Time delay 3) using pseudorandom. Based on above discussion Wi-Fi standard can be better option for Low-range Inter-satellite communication. For inter-satellite communication it is important to develop robust system for data-communication and Ranging. Wi-Fi devices with directional antenna could be used for short distance links since Wi-Fi is more establish standard and due to authentication at different layers interference could be minimized.

\section{REFERENCES}

[1] Tanya Vladimirova, Christopher P. Bridges, George Prassinos, Xiaofeng Wu, Kawsu Sidibeh, David J. Barnhart," Characterising Wireless Sensor Motes for Space Applications" In Second NASA/ESA Conference on Adaptive Hardware and Systems(AHS 2007).

[2] Daqing Gu, Jinyun Zhang," A New Measurement-Based Admission Control Method for IEEE802.11 Wireless Local Area Networks" In The 14th IEEE International Symposium on PersonalJndoor and Mobile Radio Communication Proceedings, 2003.

[3] Muhammad Owais Khan and Lili Qiu,"Accurate WiFi Packet Delivery Rate Estimation and Applications" In IEEE INFOCOM 2016 - The 35th Annual IEEE International Conference on Computer Communications.

[4] Jan Friedrich, Simon Frohn, Sascha Gübner, and Christoph Lindemann,"Understanding IEEE 802.11n Multi-hop Communication in Wireless Networks" In International Workshop on Wireless Network Measurement, 2011.

[5] Selvam T and Srikanth S,"A Frame Aggregation Scheduler for IEEE 802.11n" National Conference on Communications (NCC), 2010.

[6] Wenlong Shen, Bo Yin, Xianghui Cao, Lin X. Cai, and Yu Cheng," Secure Device-to-Device Communications over WiFi Direct" In IEEE Network (Volume: 30, Issue: 5, September-October 2016)

[7] Daniel Camps-Mur, Andres Garcia-Saavedra and Pablo Serrano," Device to device communications with WiFi Direct: overview and experimentation" In IEEE Wireless Communications (Volume: 20, Issue: 3, June 2013).

[8] F. Bouchereau and D. Brady, "Bounds on rangeresolution degradation use RSSI measurements," in 2004 IEEE International Conference on Communications (IEEE Cat. No.04CH37577), Paris, France.

[9] Steve Glass, Vallipuran Muthukkumarasamy and Marius Portmann, "The Insecurity of Time-of-Arrival DistanceRanging in IEEE 802.11 Wireless Networks", IEEE $30^{\text {th }}$ International Conference on Distributed Computing Systems Workshops (2010).

[10] https://www.education.psu.edu/natureofgeoinfo/c5_p18 